\title{
Prediction of Outcome in Diabetic Acute Ischemic Stroke Patients: A Hospital-Based Pilot Study Report
}

\author{
Amit R. Nayak ${ }^{a}$ Shweta R. Badar ${ }^{b}$ Neha Lande ${ }^{a}$ Anuja P. Kawle ${ }^{a}$ \\ Dinesh P. Kabra ${ }^{a}$ Nitin H. Chandak ${ }^{a}$ Dhananjay V. Raje ${ }^{b}$ Lokendra R. Singh $^{a}$ \\ Hatim F. Daginawala ${ }^{a}$ Rajpal S. Kashyap ${ }^{a}$ \\ aBiochemistry Research Centre, Central India Institute of Medical Sciences, Bajaj Nagar, and b MDS Bioanalytics Pvt. Ltd., \\ Sakar Enclave, Nagpur, India
}

\section{Key Words}

Diabetes mellitus · Acute ischemic stroke .

Biomarkers $\cdot$ Neuron-specific enolase $\cdot$ Inter-alpha-trypsin inhibitor heavy chain 4 protein

\begin{abstract}
Background: Demographic and clinical characteristics are known to influence the outcome in acute ischemic stroke (AIS) patients. Purpose: This study is aimed at evaluating short- and long-term outcomes in diabetic AIS patients. In addition, the study also evaluates the impact of diabetes on the performance of indigenously reported biomarker, interalpha-trypsin inhibitor heavy chain 4 (ITIH4) and known biomarkers, neuron-specific enolase (NSE) and glial-derived

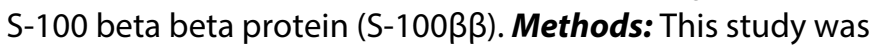
performed on 29 diabetes and 75 non-diabetes AIS patients. Outcome of AIS patients was analyzed by using modified Rankin scale at discharge, then at 12 and 18 months after discharge. Based on the obtained scores, patients were classified as improved group (scales 1-3) and dependent/expired group (scales 3-6). Blood samples were collected during admission and at discharge/expired time. Levels of NSE, S100ßß, and ITIH4 were analyzed in all samples. Results: On discharge, frequencies of dependent/expired outcome were
\end{abstract}

\section{KARGER}

E-Mail karger@karger.com

www.karger.com/aon
4/29 (14\%) and 19/75 (17\%) in diabetic and non-diabetic AIS patients. However, follow-up outcome at 12 and 18 months showed higher dependent/expired cases of 43 and $41 \%$ among diabetic AIS patients compared to 27 and $21 \%$ in non-diabetic patients. Multivariate analysis revealed that diabetes is an independent risk factor for dependent/expired outcome in AIS patients (OR 0.484 (at discharge); 1.307 (at 12 months) and 1.675 (at 18 months)). NSE, S100ßß, and ITIH4 showed a differential expression in both the outcome groups of AIS patients, irrespective of diabetes. Conclusion: Diabetes increases the risk of dependent/expired outcome in AIS patients. Also, serum NSE, S100 $\beta \beta$, and ITIH4 are independent biomarkers for prognosis of outcome in AIS patients, irrespective of diabetes.

๑ 2016 S. Karger AG, Basel

\section{Introduction}

Stroke continues to be one of the most devastating disorders worldwide, in terms of morbidity and mortality [1]. Association of comorbidity factors increases severity and

A.R.N. and S.R.B. are joint first authors of the manuscript.
(C) 2016 S. Karger AG, Basel

0972-7531/16/0234-0199\$39.50/0 
chances of unfavorable outcome in stroke patients [2]. Diabetes is one of the vital comorbidity risk factor reported to be associated with occurrence, poor outcome, and recurrence in stroke patients [3-5]. An increased incidence of stroke has been reported in advanced age among diabetic patients [6]. Recent report by the International Diabetes Federation suggested that diabetes is rapidly increasing worldwide, and it is estimated to reach a total of approximately 366 million by 2030 [7]. Moreover, Asian countries such as India and China were reported to contribute to approximately half of the world's diabetic population [8]. Despite being one of the high burden countries with a large number of diabetic patients, there is a paucity of Indian data regarding the association of diabetes and stroke in relation to stroke severity and outcome.

In the last two decades, numerous biomarkers of central nervous system (CNS) and non-CNS origins have been evaluated in the body fluids of acute ischemic stroke (AIS) patients for studying its diagnostic and prognostic efficacy [9]. Some of them such as neuron-specific enolase (NSE), S100 $\beta \beta$ protein, glial fibrillary acidic protein, myelin basic protein, creatine kinase isoenzyme $\mathrm{BB}$, tau protein, matrix metalloproteinase- 9 were found to be very promising in predicting outcomes in AIS patients $[10,11]$. In an earlier study, we reported that the serum level of Inter-alpha-trypsin inhibitor heavy chain 4 (ITIH4) protein $(120-\mathrm{kDa})$ could be a useful biomarker for prognosis of AIS patients [12,13]. Regardless of this, there are very limited studies for evaluating the impact of comorbidity risk factors of AIS on the diagnostic and prognostic potential of these biomarkers. Such information will increase the possibilities of using these biomarkers in clinical practice.

Therefore, the current hospital-based study was conducted with two objectives. The first objective was to evaluate the impact of diabetes on short- and long-term outcomes of AIS. And the second objective was to evaluate the performance of known biomarkers NSE, S-100 $\beta \beta$, and our indigenously reported biomarker ITIH4 for the prognosis of outcome in diabetic and non-diabetic AIS patients.

\section{Methods}

\section{Ethical Statement}

The study was approved by the Institutional Ethics Committee of Central India Institute of Medical Sciences (CIIMS; No. CIIMS Res/Stroke study/2011; dated September 1, 2011), Nagpur. Informed consents were obtained from all the enrolled participants and their kin for the study.

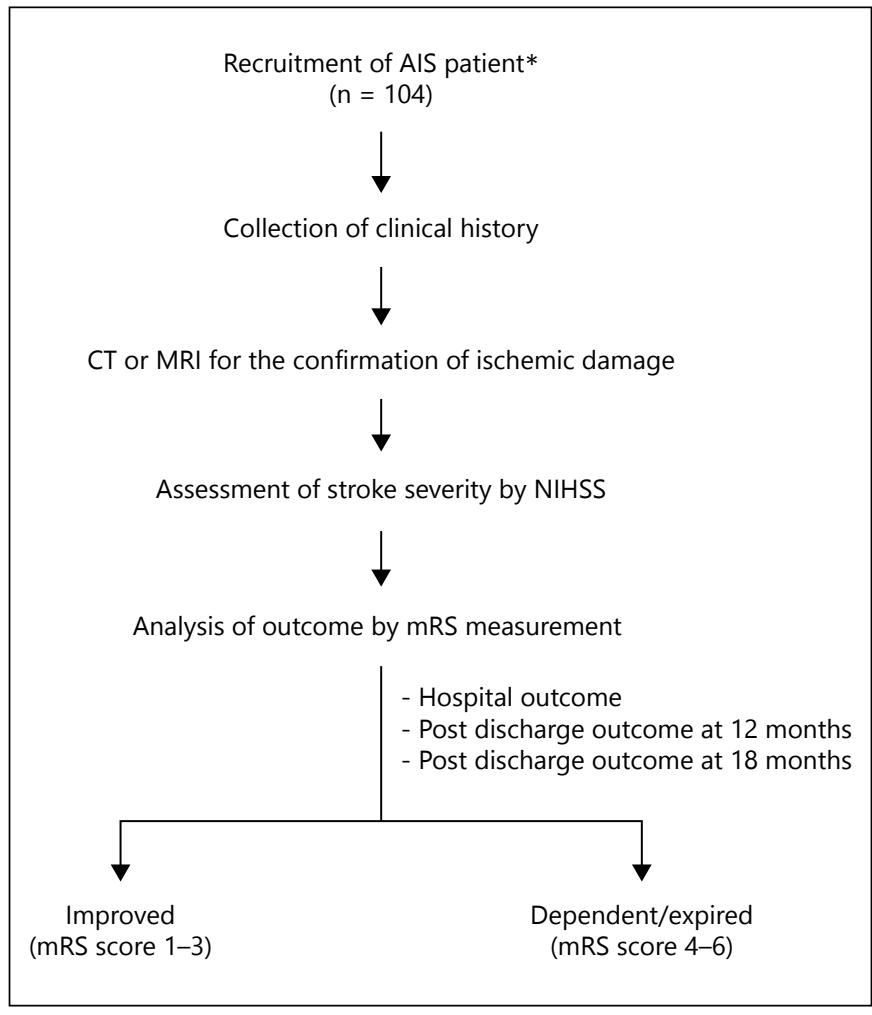

Fig. 1. Study flow chart of experimental design. * Patients who suffered hemorrhage stroke, brain malignancies, transient ischemic attacks, patients who underwent brain operations, patients who presented with severe systemic disease, dementia, psychiatric disease, active infection, patients who refused to participate in the study and patients who took discharge against medical advice were excluded from the study.

\section{Patients}

This study was carried out in a total of 104 subjects. All the subjects were selected from the inpatient department wards and intensive care unit (ICU) of CIIMS, Nagpur, India.

\section{Diagnostic Criterion}

Diagnosis of AIS was based on the WHO definition of stroke, such as 'rapidly developing signs of focal (or global) disturbance of cerebral function lasting $>24 \mathrm{~h}$ (unless interrupted by surgery or death), with no apparent non-vascular cause, history, neurological examination and CT [14]'. A patient was confirmed for diabetes if he or she had a known history of type 2 diabetes mellitus (DM), and glucose levels were $>110 \mathrm{mg} / \mathrm{dl}$. In case of those without known DM, the admission blood glucose was $\geq 150 \mathrm{mg} / \mathrm{dl}$ (fig. 1).

Selected Patients Were Subjected to the Following Protocol

Detailed history; CT scan within $12 \mathrm{~h}$ of admission to exclude non-AIS patients; severity of stroke were evaluated using the National Institute of Health Stroke Scale (NIHSS) [15]. The NIHSS score consists of 15 items and a total score of 42 points. Score of $0=$ no stroke, $1-6=$ minor stroke, $7-18=$ moderate stroke, 19 $42=$ severe stroke. The modified Rankin Scale (mRS) was used for 


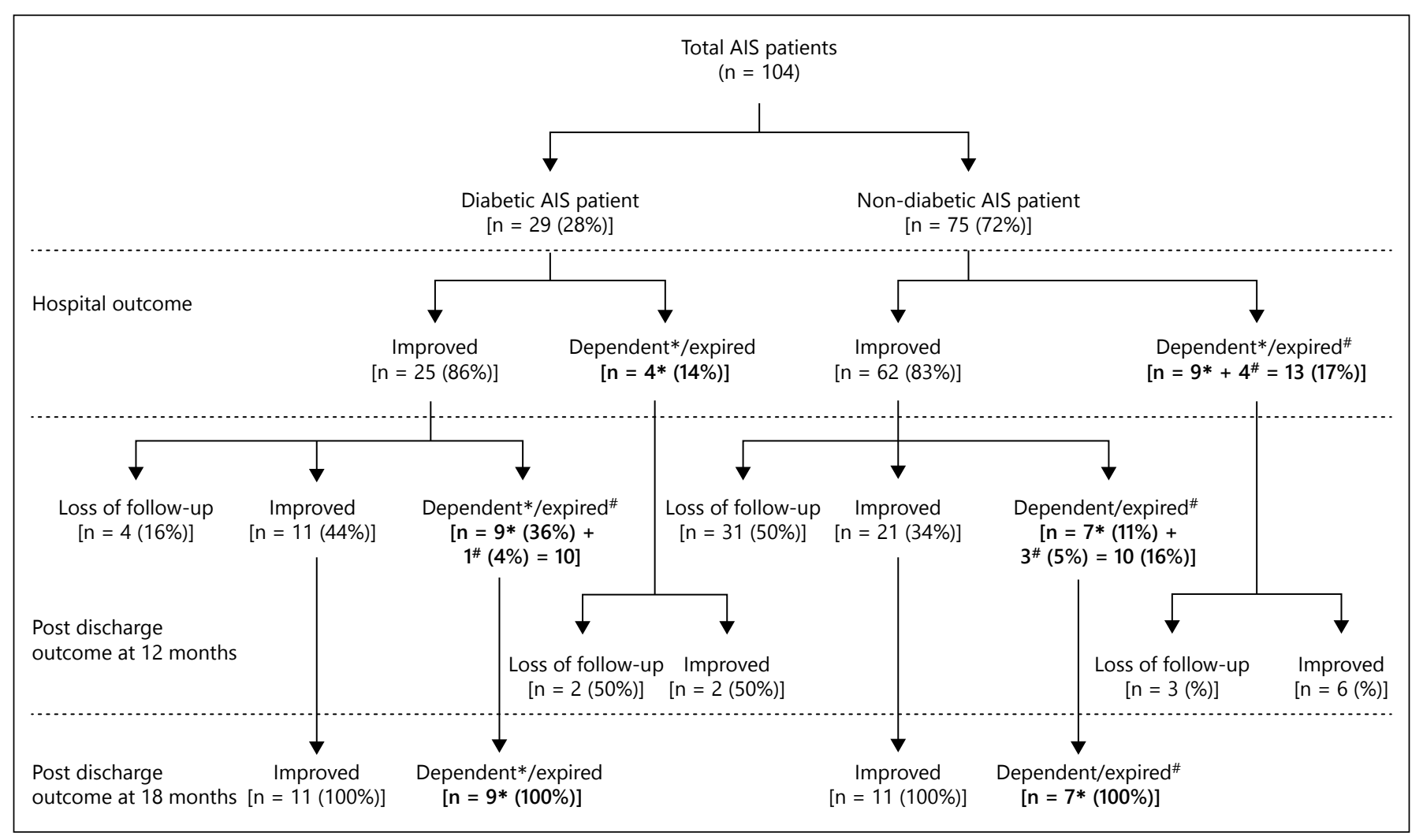

Fig. 2. Follow-up chart of AIS patients at 12 and 18 months. * Number of dependent patients; ${ }^{*}$ number of expired patients. Data in bold are summarized in table 2 .

evaluating outcome [15]. Based on mRS score, AIS patients were classified into 2 outcome groups - improved group (mRS scores 1-3) and dependent/expired group (mRS scores 4-6). All patients were admitted to the ICU and the ambient temperature was maintained between 20 to $25^{\circ} \mathrm{C}$.

\section{Exclusion Criteria}

Patients who suffered hemorrhage stroke, brain malignancies, transient ischemic attacks, patients who underwent brain operations, patients who presented with severe systemic disease, dementia, psychiatric disease, active infection, patients who refused to participate in the study and patients who took discharge against medical advice were excluded from the study.

\section{Follow-Up Study}

At 12 and 18 months after stroke onset, respectively, the followup of patients in terms of outcomes were assessed through telephonic conversation. Out of 104 AIS patients, 12-month follow-up was obtained for 60 AIS patients and 18-month follow-up was obtained for 56 AIS patients (fig. 2).

\section{Blood Samples}

Venous blood was collected from AIS patients at $0 \mathrm{~h}$ (i.e. at the time of admission), and at the time of discharge or death. Last blood sample drawn for routine investigation before the patient expired was considered as sample of expired time for the expired
AIS patient. Blood was allowed to clot, and after centrifugation ( $100 \mathrm{~g}$ for $10 \mathrm{~min}$ ), serum was separated and stored at $-20^{\circ} \mathrm{C}$ until it was used.

\section{ITIH4 Estimation Using Anti-ITIH4 Peptide Antibody}

ITIH4 was estimated using an in-house developed indirect enzyme-linked immunosorbent assay (ELISA). In brief, microtiter ELISA wells were coated with $100 \mu \mathrm{l}$ (1:400) of serum samples taken from AIS patients and blocked with $200 \mu \mathrm{l}$ of $2.5 \%$ BSA in phosphate buffer saline (PBS) for $2 \mathrm{~h}$. After washing with PBS, the polyclonal antibody against ITIH4 peptides was added (GenicBio Lab, Shanghai, China), and plates were incubated at $37^{\circ} \mathrm{C}$ for $45 \mathrm{~min}$. The wells were again washed, followed by addition of secondary antibody (goat anti-rabbit immunoglobulin horseradish peroxidase (HRP); Merck Millipore, India) and incubated for $45 \mathrm{~min}$ at $37^{\circ} \mathrm{C}$. After another wash, antibody reactivity was detected via addition of $100 \mu$ tetramethylbenzidine hydrogen peroxide $\left(\mathrm{TMB} / \mathrm{H}_{2} \mathrm{O}_{2}\right)$ substrate solution to the wells, which were then incubated at room temperature for about $5 \mathrm{~min}$. The reaction was stopped with $100 \mu \mathrm{l}$ of $2.5 \mathrm{~N} \mathrm{H}_{2} \mathrm{SO}_{4}$, and the absorbance of each well was read by Readwell Touch - Automatic Elisa Plate Reader (Robonik India Pvt. Ltd.) at $450 \mathrm{~nm}$.

\section{NSE Measurement}

NSE serum levels were estimated using Can Ag NSE EIA (Fujirebio Diagnostics, Inc., USA) as per the instructions of the kit manufacturer. The test was based on solid phase, non-competi- 
Table 1. Baseline characteristics of diabetic and non-diabetic AIS patients

\begin{tabular}{|c|c|c|c|c|}
\hline Baseline characteristics & Total, n & $\begin{array}{l}\text { Diabetic AIS } \\
\text { patients, n (\%) }\end{array}$ & $\begin{array}{l}\text { Non-diabetic } \\
\text { AIS patients, } \\
\mathrm{n}(\%)\end{array}$ & $\mathrm{p}$ value \\
\hline Number of patients & 104 & 29 & 75 & \\
\hline Age, years & & & & 0.021 \\
\hline$\leq 50$ & 38 & $5(17)$ & $33(44)$ & \\
\hline$>50$ & 66 & $24(83)$ & $42(56)$ & \\
\hline Sex & & & & 0.945 \\
\hline Male & 73 & $21(72)$ & $52(69)$ & \\
\hline Female & 31 & $8(28)$ & $23(31)$ & \\
\hline Admission within $24 \mathrm{~h}$ & 48 & $12(41)$ & $36(48)$ & 0.698 \\
\hline \multicolumn{5}{|l|}{ Associated risk factor } \\
\hline Hypertensive & 70 & $26(90)$ & $44(59)$ & 0.005 \\
\hline CVE & 16 & $5(17)$ & $11(15)$ & 0.981 \\
\hline Ischemic heart diseases & 5 & $3(10)$ & $2(3)$ & 0.258 \\
\hline Cardiac disease & 3 & $1(3)$ & $2(3)$ & 0.999 \\
\hline Other disorders & 27 & $8(28)$ & $19(25)$ & 0.999 \\
\hline \multicolumn{5}{|l|}{ Behavioral factors } \\
\hline Smoking & 8 & $1(3)$ & $7(9)$ & 0.549 \\
\hline Alcohol & 11 & $5(17)$ & $6(8)$ & 0.308 \\
\hline History of stroke & 6 & $1(3)$ & $5(7)$ & 0.871 \\
\hline Pulse rate, mean $\pm S D$ & & $81.115 \pm 12.80$ & $78.522 \pm 12.916$ & 0.384 \\
\hline \multicolumn{5}{|l|}{$\mathrm{BP}, \mathrm{mm} \mathrm{Hg}$, mean $\pm \mathrm{SD}$} \\
\hline Systolic & & $155.714 \pm 26.726$ & $142.958 \pm 24.342$ & 0.033 \\
\hline Diastolic & & $88.786 \pm 10.101$ & $88.789 \pm 16.532$ & 0.999 \\
\hline Disability score on admission & & & & 0.241 \\
\hline Severe $(\mathrm{n}=14)$ & 14 & $3(10)$ & $11(14)$ & \\
\hline Moderate $(n=44)$ & 44 & $15(52)$ & $29(39)$ & \\
\hline Mild $(n=23)$ & 23 & $8(28)$ & $15(20)$ & \\
\hline
\end{tabular}

tive immunoassay consisting of 2 monoclonal antibodies (MAbs; derived from mice) directed against 2 separate antigenic determinants of NSE molecule. The MAbs binds to $\gamma$ subunit of enzyme and thereby detects both $\gamma \gamma$ and a enzymes of NSE. In brief, the method is to transfer the required number of microplate strips to a strip frame. Wash each strip once with the wash solution. Pipette $25 \mu \mathrm{l}$ of the NSE Calibrators (CAL A, B, $\mathrm{C}, \mathrm{D}$, and $\mathrm{E}$ ) and AIS patient's specimens (unknowns) into the strip wells. Incubate the plate for $1 \mathrm{~h}( \pm 10 \mathrm{~min})$ at room temperature $\left(20-25^{\circ} \mathrm{C}\right)$ with constant shaking of the plate using a microplate shaker. After incubation, aspirate and wash each strip 6 times. Add $100 \mu \mathrm{l}$ of TMB HRP-substrate to each well. Incubate for $30 \mathrm{~min}( \pm 5 \mathrm{~min})$ at room temperature with constant shaking. After incubation, add $100 \mu \mathrm{l}$ of stop solution, mix and read the absorbance at $405 \mathrm{~nm}$ in a Readwell Touch - Automatic Elisa Plate Reader (Robonik India Pvt. Ltd.) within 15 min after addition of stop solution. Levels of NSE in the healthy control is $<0.65$ $\mu \mathrm{g} / \mathrm{l}$.

\section{S100ßß Measurement}

In vitro assay for the quantitative determination of $S 100 \beta \beta$ in human serum was performed as per the instructions indicated in the manual Can Ag S100 EIA (Fujirebio Diagnostics, Inc.,
USA). This is a 2-step enzyme immunometric assay based on 2 MAbs derived from mouse specific for 2 different epitopes of $S 100 \beta \beta$. Thus, the assay determines both $S 100 \alpha \beta$ and S100 $\beta \beta$ without cross reactivity with other forms of $S 100 \beta \beta$. In summary, ELISA wells are washed once with wash solution. Fifty microliters of $S 100 \beta \beta$ calibrators and AIS patient's sample is pipetted into the strip wells. One hundred microliters of biotin anti-S100 $\beta \beta$ is then added to each well and the frame containing the strips is incubated for $2 \mathrm{~h}( \pm 10 \mathrm{~min})$ at room temperature $\left(20-25^{\circ} \mathrm{C}\right)$ with constant shaking of the plate using a microplate shaker. After incubation, solution is aspirated and each strip is washed thrice using the wash buffer. One hundred microliters of tracer working solution is added to each well. The frame is again incubated for $1 \mathrm{~h}( \pm 5 \mathrm{~min})$ at room temperature with constant shaking. After incubation, solution is aspirated and each strip is washed 6 times. One hundred microliters of TMB HRP substrate is added to each well and incubated for 30 $\min ( \pm 5 \mathrm{~min})$ at room temperature with constant shaking. Finally, $100 \mu \mathrm{l}$ of stop solution is added to each well and mixed, and absorbance is read at $405 \mathrm{~nm}$ in a Readwell Touch - Automatic Elisa Plate Reader (Robonik India Pvt. Ltd.) within 15 min after addition of stop solution. Levels of S-100 in the healthy control are $54-90 \mu \mathrm{g} / \mathrm{l}$. 
Table 2. Assessment of outcomes during hospital discharge, and at 12 and 18 months follow-ups after discharge in diabetic and non-diabetic AIS patients

\begin{tabular}{|c|c|c|c|}
\hline & Diabetic AIS patients & Non-diabetic AIS patients & $\mathrm{p}$ value \\
\hline Total, $\mathrm{n}$ & 29 & 75 & \\
\hline Hospital stay, days & & & 0.2981 \\
\hline$\leq 15(\mathrm{n}=87)$ & $22(76)$ & $65(87)$ & \\
\hline$>15(\mathrm{n}=17)$ & $7(24)$ & $10(13)$ & \\
\hline \multicolumn{4}{|l|}{ Outcome at discharge } \\
\hline Dependent/expired $(n=17)$ & $4(14)$ & $13(17)$ & 0.442 \\
\hline \multicolumn{4}{|l|}{ Long-term outcome } \\
\hline At 12 months, $\mathrm{n}$ & $23^{*}$ & $37^{\dagger}$ & \\
\hline Dependent/expired $(n=20)$ & $10(43)$ & $10(27)$ & 0.512 \\
\hline At 18 months, $\mathrm{n}$ & $22^{\ddagger}$ & $34^{* *}$ & \\
\hline Dependent/expired $(n=16)$ & $9(41)$ & $7(21)$ & 0.356 \\
\hline \multicolumn{4}{|c|}{$\begin{array}{l}\text { * Loss to follow-up }(n=6) \text {. } \\
\text { ** Loss to follow-up }(n=34) \text {; expired at } 12 \text { months }(n=3) \text {; expired at discharge }(n=4) \text {. } \\
\dagger \text { Loss to follow-up }(n=34) \text {; expired at discharge }(n=4) \text {. } \\
\text { † Loss to follow-up }(n=6) \text {; expired at } 12 \text { months }(n=1) \text {. }\end{array}$} \\
\hline
\end{tabular}

\section{Statistical Analysis}

The categorical data on different baseline variables was expressed in terms of number and percentage, and its association with study groups was determined using the chi-square test. The data on continuous variables was summarized in terms of mean and $\mathrm{SD}$, and statistical significance of difference in the variable means between groups was determined using $t$ test for independent samples. Significance of difference in the mean levels of peptides, anti-peptides, and selected proteins between study groups was also determined using $t$ test for independent samples. Survival data on patients was used to study survival rates in study groups. Outcome was defined as either patient expired or dependent after discharge. Kaplan-Meier plots were constructed for each study group, and the statistical significance of difference between the plots was determined using log-rank test. Considering death or dependency as outcome, the association of diabetes with outcome was analyzed using multivariate logistic regression after adjusting for potential confounders such as age, sex, alcohol, thrombolysis, admission duration, and hypertension. Analysis was performed using $\mathrm{R}$ state package version 3.0.0 (R-programming language), and statistical significance was tested at $5 \%$ level.

\section{Results}

Out of 104 patients, 29 (28\%) were diabetic and 75 (72\%) were non-diabetic. Baseline characteristics associated with diabetic and non-diabetic AIS patients are given in table 1 . We found that $83 \%$ of diabetic AIS patients belonged to the old age group, that is, above 50 years with a higher proportion of males $(72 \% ; \mathrm{p}=0.0020)$. Similarly, hypertension was found to be significantly associated with $90 \%$ of diabetic AIS patients $(\mathrm{p}=0.005)$. This was also very much observable in systolic blood pressure (BP; $\mathrm{p}=0.003$ ).

Results of assessment of short-term outcome during hospital stay and long-term outcome at 12 and 18 months after discharge in diabetic and non-diabetic AIS patients are given in table 2 . We found that the frequency of death or dependency in diabetic AIS patients was very much similar to that of non-diabetic AIS patients in hospital. However, in the follow-ups taken at 12 and 18 months, the frequency of death or dependency was found to be higher ( $p>0.05$ ) in diabetic AIS patients (i.e. 43 and $41 \%$ ) than non-diabetic AIS patients (i.e. 27 and 21\%).

When multivariate logistic-regression analysis was performed by adjusting for potential confounders such as age, sex, hypertension, smoking, alcohol, admission within $24 \mathrm{~h}$, thrombolysis (table 3 ), it was observed that diabetes was an independent risk factor for death or dependency at 12 months (OR 1.307; 95\% CI 0.372-4.586), and at 18 months (OR 1.675; 95\% CI 0.438-6.412) after hospital discharge. A survival curve (log-rank analyses) for mortality in the evaluated AIS patients was prepared to study the impact of diabetes on the survival of AIS patients. We found a higher survival in non-diabetic AIS patients compared to diabetic AIS patients, as shown in online supplementary figure 1 (see www.karger.com/ doi/10.1159/000449480). However, the difference was statistically non-significant. 
Table 3. Unadjusted and adjusted ORs of poor outcomes in diabetic AIS patient compared with non-diabetic AIS patients

\begin{tabular}{|c|c|c|}
\hline Outcome & Unadjusted OR (95\% CI) & Adjusted OR* $(95 \% \mathrm{CI})$ \\
\hline \multicolumn{3}{|l|}{ Outcome at discharge } \\
\hline Dependent/expired $(n=17)$ & $0.78(0.19-2.49)$ & $0.484(0.053-4.44)$ \\
\hline \multicolumn{3}{|l|}{ Long-term outcome } \\
\hline \multicolumn{3}{|l|}{ At 12 months } \\
\hline Dependent/expired $(n=20)$ & $2.04(0.67-6.34)$ & $1.307(0.372-4.586)$ \\
\hline \multicolumn{3}{|l|}{ At 18 months } \\
\hline Dependent/expired $(n=16)$ & $2.60(0.78-9.05)$ & $1.675(0.438-6.412)$ \\
\hline
\end{tabular}

Results of the estimation of serum biomarker (i.e. ITIH4, NSE, and S100 $\beta \beta$ ) in diabetic and non-diabetic AIS patients with improved and dependent/expired outcomes are given in figures 3 and 4 , respectively. We found that ITIH4 levels detected by anti-peptides 2, 6, 7 increases significantly $(p<0.05)$ at discharge time (vs. Admission) among the AIS patients with an improved hospital outcome (fig. 3). But in AIS patients with dependent/expired outcome, the level of ITIH4 at the time of discharge either remains stable or decreases further compared to the level at admission. Other anti-peptides (i.e. 1, 3, 4, 5, 8 and 9) did not show any differential expression pattern in improved and dependent/expired AIS patients with and without diabetes, respectively (data not shown). Similarly, results of NSE and S100 $\beta \beta$ (fig. 4) show that their levels are upregulated at the time of discharge or expiry in diabetic AIS patients with dependent/expired outcome ( $\mathrm{p}<0.05$ vs. admission value). Also, non-diabetic AIS patients showed a similar type of results, but were statistically non-significant.

\section{Discussion}

In this hospital-based pilot study, we evaluated the impact of diabetes on short- and long-term outcomes of AIS. We found that both diabetic and non-diabetic patients had a nearly similar hospital outcome; the results of 12 and 18 months outcome showed a higher number of dependent/expired cases in diabetic AIS patients.

Diabetes is known to increase the risk of stroke and worsen the clinical outcome in stroke patients. However, the exact mechanism by which diabetes induces stroke is unclear till date $[16,17]$. Similarly, risk of diabetes and stroke were reported to increase with advancing age [6,
18]. We found that majority of diabetic AIS patients (83\%) were of advanced age group (i.e. $>50$ years). Furthermore, we also found an estimated $28 \%$ diabetic AIS patients and $72 \%$ non-diabetic AIS patients in our study population. Kissela et al. [19] reported an estimated 37$42 \%$ stroke cases with diabetes in both African and Americans. Thus, incidences of stroke patients with diabetes are less in India as compared to Africans and Americans. However, India and China are reported to contribute to approximately half of the world's diabetic population [8]. This suggests that India is at risk of having higher number of diabetic stroke patients in the future.

Meta-analysis for studying the association of sex of stroke patients and diabetes suggested that the risk of stroke associated with diabetes is significantly more common in women than men [20]. Tuomilehto et al. [18] reported that female diabetic stroke patients have a higher risk of death than males, while a study conducted on Indian population suggests that there is no sex difference in the prevalence of diabetes in Indian population [21]. In contrast to earlier reports, we observed a higher ratio of male diabetic AIS patients in our study population. This suggests a regional difference in the prevalence of diabetic stroke patients and warrants further study to confirm findings and associated risk factors which are responsible for this differential observation.

Similarly, hypertension is reported to be more common among diabetic AIS patients compared to non-diabetic AIS patients [22]. Coexistence of diabetes and hypertension is also reported to be a major contributor for the poor health outcome [23]. Furthermore, systolic hypertension is known to be an important predictor of death in patients suffering from coronary heart disease, stroke, cardiovascular disease [24] Similarly, an increase in the systolic BP is also reported to increase the risk of compli- 


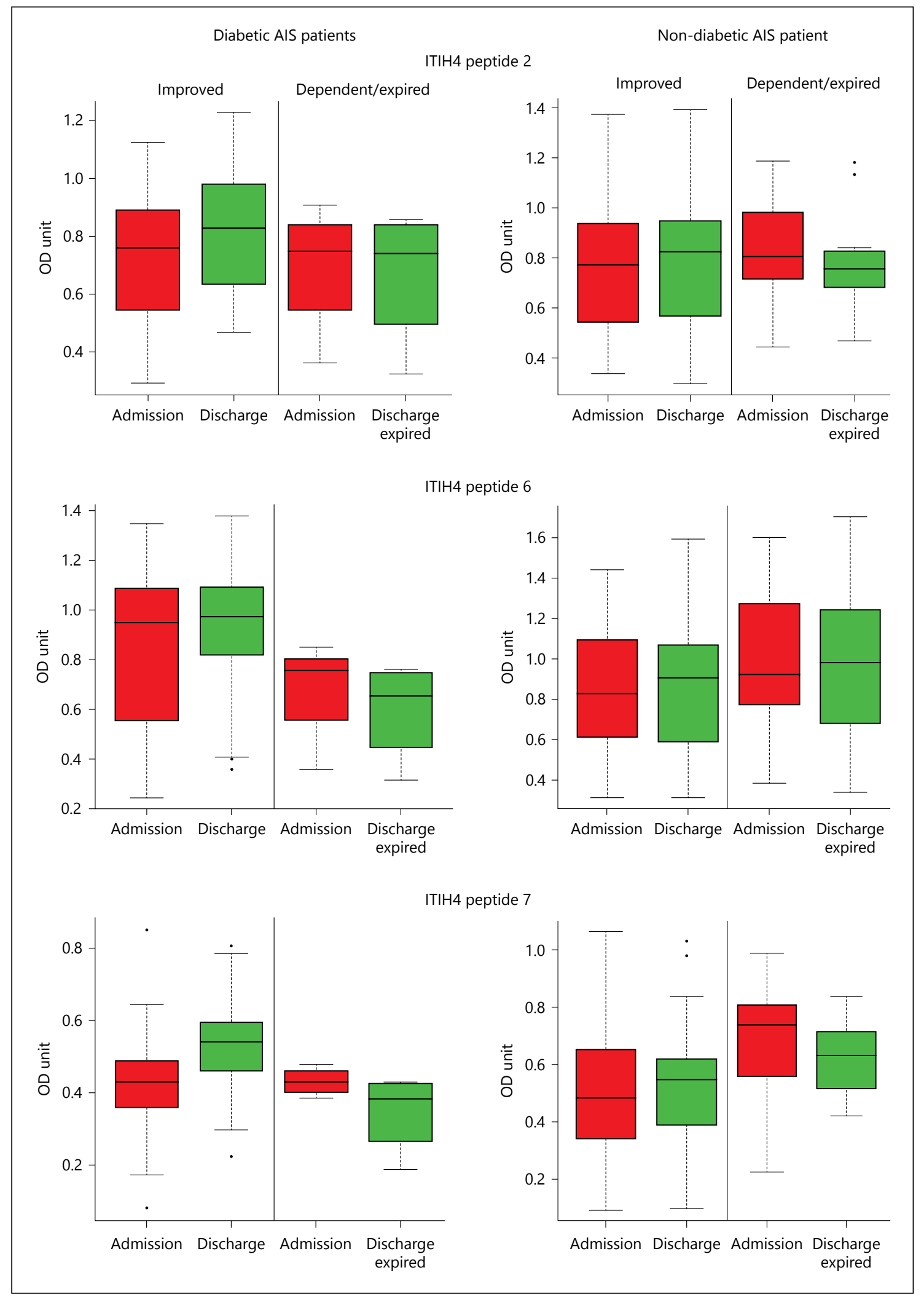

Fig. 3. Level of ITIH4 in diabetic $(n=29)$ and non-diabetic $(n=75)$ AIS patients with improved and dependent/ expired outcomes. 


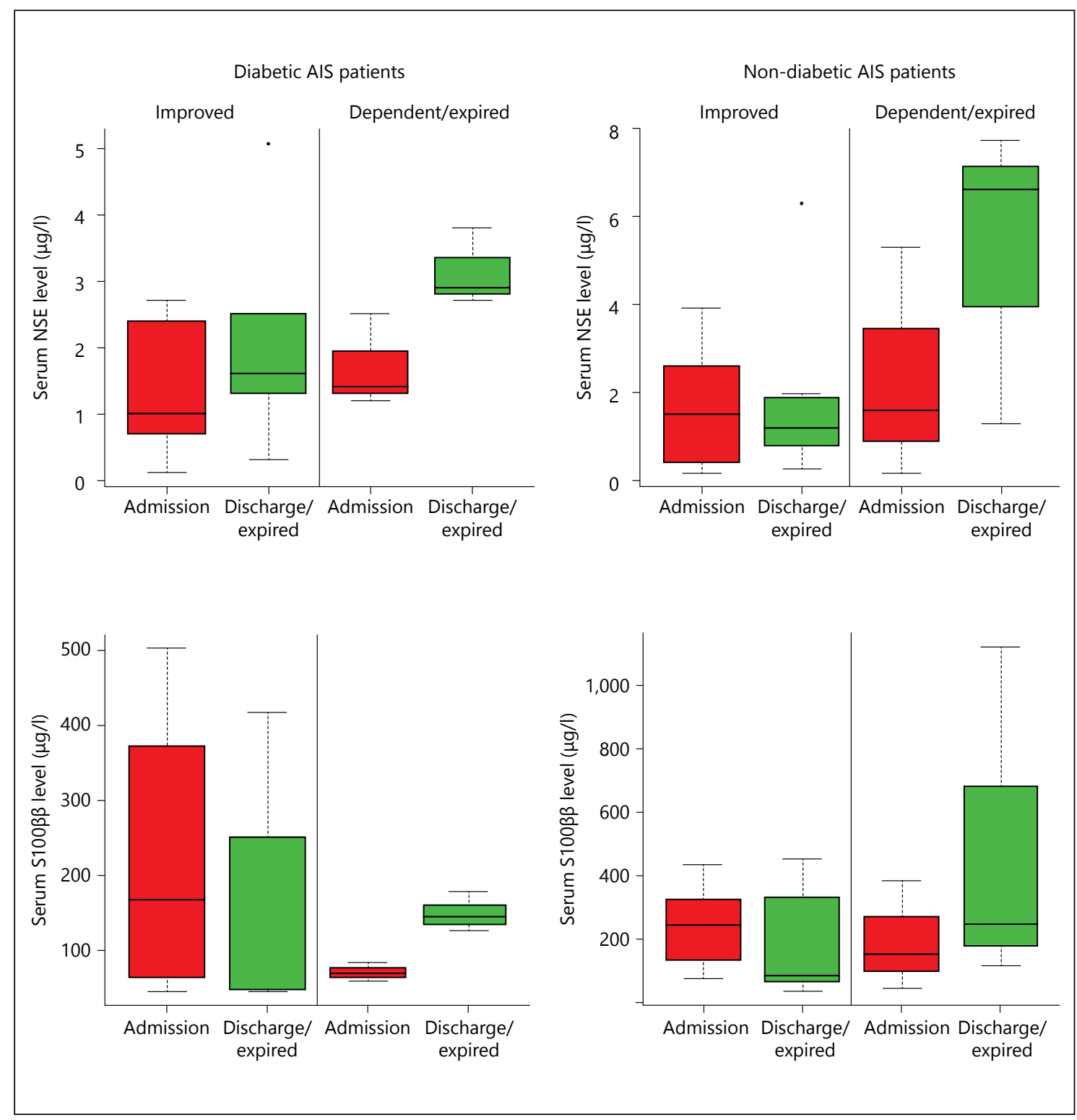

Fig. 4. Levels of NSE and S100 $\beta$ in diabetic $(n=29)$ and non-diabetic $(n=75)$ AIS patients with improved and dependent/expired outcomes.

cations in diabetic patients [25]. Control of BP among diabetic patients is known to reduce the risk of diabetesrelated complications [26, 27]. We observed hypertension in most of the diabetic AIS patients (i.e. 90\%) compared to non-diabetic AIS patients (i.e. 59\%). Likewise, diabetic AIS patients had a higher systolic BP (BP $>150$ $\mathrm{mm} \mathrm{Hg}$ ) compared to non-diabetic AIS patients (i.e. BP $142 \mathrm{~mm} \mathrm{Hg}$ ). Furthermore, systolic hypertension is one of the major risk factor associated with diabetic AIS patients. Thus, poor outcome in diabetic AIS patients at 12 and 18 months after hospital discharge could possibly be due to a higher number of systolic hypertensive patients in this group. Therefore, management of diabetic and systolic BP is vital for the better outcome in diabetic AIS patients.

In addition to the evaluation of impact of diabetes on the outcome of AIS patients, we also evaluated the performance of our indigenously reported biomarker ITIH4 in predicting the outcome in diabetic and non-diabetic AIS patients in comparison with standard biomarkers NSE, 
S-100 $\beta \beta$. ITIH4 is an inter-alpha trypsin heavy chain-related protein. It inhibits the phagocyte activities of polymorph nuclear cells and may act as an anti-inflammatory protein. In our earlier studies, we have reported that the expression of ITIH4 downregulates in AIS patients, and further it is normalized only in those patients whose health improved and not in those who either expired or their health deteriorated. Therefore, it could be a novel marker for prognosis of AIS [12]. Thus, an increase in ITIH4 level in the follow-up serum sample of AIS patients suggests sustained anti-inflammatory activity [28]. In this study, we found that ITIH4 level estimated using antiITIH4 peptides 2, 6 and 7 showed an increase in expression of ITIH4 in the follow-up serum samples of improved AIS patients collected at discharge but not in dependent or expired AIS patients irrespective of diabetes. This shows that peptides 2,6 , and 7 are the only immunodominant and specific peptide sequence of ITIH4. Therefore, estimation of serum ITIH4 peptides 2, 6, and 7 can be an important marker for the prognosis of AIS. Similarly, NSE is a soluble protein enolase enzyme found predominantly in neurons and neuroendocrine cells [29]. $S 100 \beta \beta$ proteins are a family of low-molecular-weight calcium-binding protein found in glial cells [30]. Normally, NSE is present only in negligible amounts in the peripheral blood. Elevated NSE levels were reported within $4 \mathrm{~h}$ of stroke onset [31]. It has been reported to be an indicator of infarction volume and worse neurological outcome after ischemic stroke [32]. Serum S100 $\beta \beta$ protein level was known to increase after $8 \mathrm{~h}$ of onset of stroke and also correlate with severity and functional outcome in stroke patients [33,34]. We also observed higher value of NSE and S100 $\beta \beta$ both in diabetic and non-diabetic AIS patients, with a dependent or expiry outcome. This suggests that ITIH4, NSE, and S100 $\beta \beta$ are independent biomarkers for the prognosis of AIS patients.
The major limitation of our study is less number of samples to represent the Indian population. Similarly, another limitation associated with the study is the low number of follow-up data on AIS patients' clinical status at 12 and 18 months after hospital discharge. However, the findings obtained were very encouraging to conduct a large-scale study.

Based on the observations, it can be concluded that diabetes increased the risk of dependent/expired outcome in AIS patients. Age and hypertension were found to be major risk factors for the dependent/expired outcome among diabetic AIS patients. Serum NSE, S100 $\beta \beta$, and ITIH4 levels could predict the outcome of AIS patients, irrespective of their diabetic and non-diabetic conditions.

\section{Acknowledgment}

All the authors would like to acknowledge Department of Biotechnology, India for funding this project (project No. BT/ PR14368/MED/30/525/2010).

\section{Authorship Contribution}

A.R.N. and S.R.B.: article drafting, conception and design and analysis and interpretation of data; N.L. and A.P.K.: article drafting and data collection; D.P.K. and N.H.C.: analysis and interpretation of data; D.V.R.: statistical analysis; L.R.S. and H.F.D.: final approval of the article; R.S.K.: conception and design, critical revision of the article and overall responsibility.

\section{Disclosure Statements}

The authors declare no conflict of interest. The manuscript is complied with International Committee of Medical Journal Editor's guidelines.

\section{References}

1 Mathers CD, Lopez A, Stein C, Murray CJL: Deaths and Disease Burden by Cause: Global Burden of Disease Estimates for 2001 by World Bank Country Groups. Bethesda, Disease Control Priorities Project Working Paper 18.

2 Karatepe AG, Gunaydin R, Kaya T, Turkmen G: Comorbidity in patients after stroke: impact on functional outcome. J Rehabil Med 2008;40:831-835.

3 Stead LG, Gilmore RM, Bellolio MF, Mishra S, Bhagra A, Vaidyanathan L, Decker WW, Brown RD Jr: Hyperglycemia as an independent predictor of worse outcome in non-dia- betic patients presenting with acute ischemic stroke. Neurocrit Care 2009;10:181-186.

4 Reeves MJ, Vaidya RS, Fonarow GC, Liang L, Smith EE, Matulonis R, Olson DM, Schwamm LH; Get with the Guidelines Steering Committee and Hospitals: Quality of care and outcomes in patients with diabetes hospitalized with ischemic stroke: findings from get with the guidelines - stroke. Stroke 2010;41:e409e417.

5 Jia Q, Zhao X, Wang C, Wang Y, Yan Y, Li H, Zhong L, Liu L, Zheng H, Zhou Y, Wang Y: Diabetes and poor outcomes within 6 months after acute ischemic stroke: the China national stroke registry. Stroke 2011;42:2758-2762.

6 Policardo L, Seghieri G, Francesconi P, Anichini R, Franconi F, Seghieri C, Del Prato S: Gender difference in diabetes-associated risk of first-ever and recurrent ischemic stroke. J Diabetes Complications 2015;29:713-717.

7 http://www.who.int/diabetes/actionnow/en/ DANbooklet.pdf (cited December 2014).

8 Umemura T, Kawamura T: Effect of diabetes on stroke symptoms and mortality: lessons from a recent large population-based cohort study. J Diabetes Investig 2014;5:14-16. 
9 Barr TL, Matarin MD, Warch JS, Singleton $\mathrm{AB}$, Conley YP: Biomarkers for Acute Ischemic Stroke, 2013, Application No. 13/580571, United States Patent Application 20130189243, Kind Code: A1.

10 Els T, Bruckmann J, Röhn G, Daffertshofer M, Mönting JS, Ernestus RI, Hennerici M: Spermidine: a predictor for neurological outcome and infarct size in focal cerebral ischemia? Stroke 2001;32:43-46.

11 Purrucker JC, Herrmann O, Lutsch JK, Zorn M, Schwaninger M, Bruckner T, Auffarth GU, Veltkamp R: Serum protein $\mathrm{S} 100 \beta$ is a diagnostic biomarker for distinguishing posterior circulation stroke from vertigo of nonvascular causes. Eur Neurol 2014;72:278284.

12 Kashyap RS, Nayak AR, Deshpande PS, Kabra D, Purohit HJ, Taori GM, Daginawala HF: Inter-alpha-trypsin inhibitor heavy chain 4 is a novel marker of acute ischemic stroke. Clin Chim Acta 2009;402:160-163.

13 Nayak AR, Kashyap RS, Kabra D, Purohit HJ, Taori GM, Daginawala HF: Time course of inflammatory cytokines in acute ischemic stroke patients and their relation to inter-alfa trypsin inhibitor heavy chain 4 and outcome. Ann Indian Acad Neurol 2012;15:181-185.

14 Aho K, Harmsen P, Hatano S, Marquardsen J, Smirnov VE, Strasser T: Cerebrovascular disease in the community: results of a WHO collaborative study. Bull World Health Organ 1980;58:113-130.

15 Harrison JK, McArthur KS, Quinn TJ: Assessment scales in stroke: clinimetric and clinical considerations. Clin Interv Aging 2013;8: 201-211.

16 Barrett-Connor E, Khaw KT: Diabetes mellitus: an independent risk factor for stroke? Am J Epidemiol 1988;128:116-123.

17 Air EL, Kissela BM: Diabetes, the metabolic syndrome, and ischemic stroke: epidemiology and possible mechanisms. Diabetes Care 2007;30:3131-3140.
18 Tuomilehto J, Rastenyte D, Jousilahti P: Diabetes mellitus as a risk factor for death from stroke. Prospective study of the middle-aged Finnish population. Stroke 1996;27:210-215.

19 Kissela BM, Khoury J, Kleindorfer D, Woo D, Schneider A, Alwell K, Miller R, Ewing I, Moomaw CJ, Szaflarski JP, Gebel J, Shukla R, Broderick JP: Epidemiology of ischemic stroke in patients with diabetes: the greater cincinnati/northern Kentucky stroke study. Diabetes Care 2005;28:355-359.

20 Peters SA, Huxley RR, Woodward M: Diabetes as a risk factor for stroke in women compared with men: a systematic review and meta-analysis of 64 cohorts, including 775,385 individuals and 12,539 strokes. Lancet 2014; 383:1973-1980

21 Qiao Q, Hu G, Tuomilehto J, Nakagami T, Balkau B, Borch-Johnsen K, et al; DECODA Study Group: Age- and sex-specific prevalence of diabetes and impaired glucose regulation in 11 Asian cohorts. Diabetes Care 2003; 26:1770-1780.

22 Klein R, Klein BE, Lee KE, Cruickshanks KJ, Moss SE: The incidence of hypertension in insulin-dependent diabetes. Arch Intern Med 1996; 156:622-627.

23 Codreanu I, Sali V, Gaibu S, Suveica L, Popa S, Perico N, Ene-Iordache B, Carminati S, Feehally J, Remuzzi G: Prevalence of hypertension and diabetes and coexistence of chronic kidney disease and cardiovascular risk in the population of the republic of moldova. Int J Hypertens 2012;2012:951734.

24 Antikainen R, Jousilahti P, Tuomilehto J: Systolic blood pressure, isolated systolic hypertension and risk of coronary heart disease, strokes, cardiovascular disease and all-cause mortality in the middle-aged population. J Hypertens 1998;16:577-583.

25 Adler AI, Stratton IM, Neil HA, Yudkin JS, Matthews DR, Cull CA, Wright AD, Turner RC, Holman RR: Association of systolic blood pressure with macrovascular and microvascular complications of type 2 diabetes (UKPDS 36): prospective observational study. BMJ 2000;321:412-419.
26 Arauz-Pacheco C, Parrott MA, Raskin P; American Diabetes Association: Treatment of hypertension in adults with diabetes. Diabetes Care 2003;26(suppl 1):S80-S82.

27 Cooper ME, Johnston CI: Optimizing treatment of hypertension in patients with diabetes. JAMA 2000;283:3177-3179.

28 Ahmad O, Wardlaw J, Whiteley WN: Correlation of levels of neuronal and glial markers with radiological measures of infarct volume in ischaemic stroke: a systematic review. Cerebrovasc Dis 2012;33:47-54.

29 Pandey A, Saxena K, Verma M, Bharosay A: Correlative study between neuron-specific enolase and blood sugar level in ischemic stroke patients. J Neurosci Rural Pract 2011; 2:50-54.

30 Marenholz I, Heizmann CW, Fritz G: S100 proteins in mouse and man: from evolution to function and pathology (including an update of the nomenclature). Biochem Biophys Res Commun 2004;322:1111-1122.

31 Stevens H, Jakobs C, de Jager AE, Cunningham RT, Korf J: Neurone-specific enolase and $\mathrm{N}$-acetyl-aspartate as potential peripheral markers of ischaemic stroke. Eur J Clin Invest 1999;29:6-11.

32 Cunningham RT, Watt $M$, Winder J, McKinstry S, Lawson JT, Johnston CF, Hawkins SA, Buchanan KD: Serum neuronespecific enolase as an indicator of stroke volume. Eur J Clin Invest 1996;26:298-303.

33 Fassbender K, Schmidt R, Schreiner A, Fatar M, Mühlhauser F, Daffertshofer M, Hennerici M: Leakage of brain-originated proteins in peripheral blood: temporal profile and diagnostic value in early ischemic stroke. J Neurol Sci 1997;148:101-105.

34 Foerch C, Otto B, Singer OC, Neumann-Haefelin T, Yan B, Berkefeld J, Steinmetz H, Sitzer M: Serum S100B predicts a malignant course of infarction in patients with acute middle cerebral artery occlusion. Stroke 2004;35:21602164. 